\title{
Caracterización del conocimiento didáctico-matemático de futuros profesores de matemáticas de secundaria
}

\author{
Eduardo Mendoza Quintero \\ Ana Guadalupe Del Castillo Bojórquez \\ Universidad de Sonora \\ e-mail: edumenqui@gmail.com
}

\begin{abstract}
Resumen
En este artículo se presentan los avances de un trabajo de investigación que se está realizando, el cual se corresponde con un proyecto de tesis de Maestría en Ciencias con Especialidad en Matemática Educativa de la Universidad de Sonora. Con éste se pretende caracterizar el conocimiento didáctico-matemático que ponen en juego futuros docentes de matemáticas de secundaria durante su Fase de Prácticas correspondiente al último año de formación inicial en una Escuela Normal Superior (ENS). Para lograr lo anterior, se aplican las nociones teóricas que proporciona el Enfoque Ontosemiótico del Conocimiento y la Instrucción Matemática, principalmente el Modelo del Conocimiento Didáctico-Matemático (CDM), el cual nos proporciona categorías de análisis de los conocimientos didácticos y disciplinares del profesor de matemáticas.

Aquí se presenta una parte del estudio realizado, la cual se centra en la determinación del significado institucional de referencia sobre los contenidos matemáticos estudiados, el cual será utilizado en análisis posteriores para la caracterización del conocimiento matemático del futuro profesor.
\end{abstract}

\section{Introducción}

El trabajo de investigación que se reporta consiste en un estudio de carácter descriptivo sobre los conocimientos disciplinares y didácticos de futuros profesores de matemáticas de secundaria que se forman en una ENS. Nos interesamos en su Fase de Prácticas, periodo correspondiente a su último año de formación inicial donde se encuentran a cargo de un grupo de estudiantes de nivel secundaria a lo largo de dos terceras partes del ciclo escolar. Se pretende analizar y describir los diferentes conocimientos que los normalistas ponen en juego desde la planificación que realizan para la clase, hasta el momento de su implementación en el aula. Para la realización de nuestro estudio fueron seleccionados tres estudiantes normalistas que iniciaban su último año de formación en la ENS de Hermosillo. Los contenidos matemáticos estudiados son los correspondientes al apartado "Patrones y ecuaciones" del Bloque II del Programa de Matemáticas de Tercer grado de Educación Secundaria del Plan de Estudios para la Educación Básica 2011. La observación se realizó durante el periodo de Fase de Prácticas de los normalistas.

Para la elaboración de los instrumentos de investigación, para el análisis de resultados obtenidos, la organización e interpretación de los mismos, hacemos uso de los constructos teóricos del Enfoque Ontosemiótico del Conocimiento y la Instrucción Matemática. Así, 
el análisis de la información obtenida se plantea a partir del Modelo del Conocimiento Didáctico-Matemático que nos permitirá estudiar el proceso de instrucción de los futuros docentes mediante el análisis de las diferentes facetas involucradas (epistémica, cognitiva, afectiva, mediacional, interaccional, ecológica), llevándonos a caracterizar los conocimientos disciplinares y didácticos que han puesto en juego.

Se reporta una parte del análisis de la faceta epistémica, referente a los conocimientos matemáticos relativos al contexto institucional en que se realiza el proceso de estudio. Para esto determinamos el significado institucional de referencia sobre los contenidos matemáticos que los normalistas abordan durante su Fase de Prácticas. Se advierte del trabajo que resta realizar en nuestra investigación, así como la forma en que los resultados obtenidos nos llevarán a cumplir el objetivo planteado.

\section{Problema de investigación y marco referencial}

Algunos investigadores y especialistas como Shulman (1987), Hill, Ball, Schilling (2008), Shoenfeld y Kilpatrick (2008), Godino (2009), entre otros, son más o menos coincidentes en su visión acerca del conocimiento didáctico-matemático del profesor y en general se enfocan en el conocimiento del contenido y el conocimiento pedagógico del contenido. El primero referente al conocimiento del tipo matemático que es necesario para resolver problemas matemáticos y el segundo al conocimiento sobre el contenido de la enseñanza y cómo los estudiantes aprenden.

Con base en el interés por categorizar los conocimientos con los que debe contar el profesor de matemáticas, en nuestro trabajo de investigación nos hemos planteado conocer aspectos centrales de la formación inicial que reciben futuros profesores de matemáticas de secundaria en una institución destinada a esta formación, con el fin de poder contar con elementos de estudio que en un momento dado ayuden a comprender de mejor manera la problemática que se vive con relación a la enseñanza y aprendizaje de las matemáticas.

Tomando en cuenta que formalmente en nuestro país las escuelas de Educación Básica son atendidas por profesores egresados de una Escuela Normal, esto tiene como implicación que recibieron una formación pedagógica, didáctica y disciplinar, que los califica y certifica para desempeñarse como docentes (Sandoval, 2009).

En nuestro estudio consideramos a la Escuela Normal Superior de Hermosillo (ENSH), la cual ofrece la Licenciatura en Educación con Especialidad Matemáticas, donde los estudiantes que aquí se forman podrán proporcionar una muestra significativa que nos ayude a dar una idea de cómo está la formación inicial de nuestros profesores de matemáticas, ya que los egresados de aquí laborarán como docentes de matemáticas en alguna institución de educación secundaria en el Estado.

Al hablar de formación inicial nos referimos específicamente a la formación didáctica y disciplinar que reciben los normalistas. Para conocer esta formación nos interesamos en su último año en la ENSH. En este periodo se encuentran a cargo de un grupo de estudiantes de educación secundaria durante dos terceras parte del ciclo escolar, de manera que el resto del ciclo asisten a la ENSH para analizar esta experiencia y realizar trabajos académicos en 
torno a ella. "Este periodo es una forma especial de iniciarlos en la docencia" (Sandoval, 2009, p.186).

Este periodo lo identificamos como la Fase de Prácticas de los normalistas. Es durante esta etapa de su formación que el trabajo docente que desarrollan mediante la práctica intensiva, durante periodos prolongados, en grupos de secundaria, implica la realización de actividades sistemáticas de enseñanza que, a su vez, demandan la integración de los conocimientos desarrollados a lo largo de su formación inicial, tanto en el ámbito disciplinar como didáctico (Sandoval, 2009).

En este trabajo se reconoce la importancia de los conocimientos que se crean a lo largo de la formación inicial de los futuros profesores de matemáticas de secundaria, el interés se centra en la etapa correspondiente a su Fase de Prácticas descrita anteriormente, en particular: ¿Qué conocimientos disciplinares y didácticos se ponen en juego? ¿Podemos caracterizar estos conocimientos por parte del futuro profesor? Con base en estas interrogantes enunciamos nuestra pregunta de investigación de la siguiente manera:

¿Cuáles son las características del conocimiento didáctico-matemático de los futuros profesores de matemáticas de secundaria en el último año de su formación inicial?

Para poder dar respuesta a la pregunta de investigación, se estudian aspectos centrales de la Fase de Prácticas de los normalistas. Tal como se menciona, ésta demanda integrar los aprendizajes didácticos y disciplinares que se han desarrollado, por tal razón, este periodo se muestra como clave para conocer el conocimiento didáctico-matemático que se pone en juego en la realización de sus prácticas de enseñanza antes de iniciar en la docencia. Con base en el interés anterior el objetivo general de nuestro trabajo es el siguiente:

Caracterizar el conocimiento didáctico-matemático de los futuros profesores de matemáticas de secundaria que se pone en juego en su Fase de Prácticas.

El desarrollo de este trabajo podría aportar elementos de estudio que ayuden a comprender de mejor manera la problemática que se vive con relación a la enseñanza y aprendizaje de las matemáticas en secundaria, y a encaminar acciones destinadas a una formación continua para estos futuros docentes.

\subsection{Los contenidos matemáticos estudiados}

Los contenidos matemáticos abordados en el aula durante el tiempo de la Fase de Prácticas de los normalistas en que se realizó la observación, se ubican en los apartados "Patrones y ecuaciones" y "Figuras y cuerpos" del Bloque II del Programa de Matemáticas de Tercer Grado de Secundaria del Plan de estudios para la Educación Básica 2011.

Los ejes temáticos correspondientes son "Sentido numérico y pensamiento algebraico" y "Forma, espacio y medida", respectivamente. En este trabajo nos efocaremos en analizar el apartado "Patrones y ecuaciones", para el cual la propuesta curricular establece que deben abordarse los siguientes contenidos: "Uso de ecuaciones cuadráticas para modelar situaciones y resolverlas usando la factorización" (SEP, 2011, p. 47). 


\section{Consideraciones teóricas y metodológicas}

\subsection{Elementos teóricos}

Como anteriormente ha sido mencionado, el presente trabajo de investigación se sustenta en el marco teórico del Enfoque Ontosemiótico del Conocimiento y la Instrucción Matemática (EOS), el cual ha sido desarrollado por Godino y colaboradores (Godino, Batanero y Font, 2009). Las nociones teóricas que maneja el EOS las vemos como herramientas para el análisis y reflexión de los procesos de enseñanza y aprendizaje, y que pueden ser empleadas para indagar sobre las prácticas de los profesores con el propósito de comprender y analizar de manera sistemática y con distintos niveles de profundidad, los diversos aspectos implicados en la enseñanza y aprendizaje de las matemáticas.

En este trabajo se considera la determinación del significado institucional de referencia sobre los contenidos matemáticos estudiados. Esto llevará a realizar un análisis detallado de los conocimientos matemáticos que debieron ser promovidos durante el proceso de instrucción llevado a cabo por los futuros docentes, desde su planeación de clase y al momento de su implementación en el aula. En este punto se considera clave la identificación de objetos matemáticos primarios (situaciones, lenguajes, conceptos, proposiciones, procedimientos, argumentos) y las relaciones entre ellos formando configuraciones epistémicas.

Las nociones teóricas consideradas hasta aquí, son herramientas básicas que propone el EOS para explicar las distintas componentes que intervienen en la cognición matemática. Pero también consideramos su propuesta para aplicar su enfoque a los problemas de su didáctica. Así, para analizar el conocimiento didáctico-matemático de los futuros docentes, el EOS nos proporciona herramientas para el análisis de distintas facetas y niveles de este conocimiento en el Modelo del Conocimiento Didáctico-Matemático (CDM) (Godino, 2009).

Este Modelo identifica seis facetas para analizar los procesos de instrucción matemática: epistémica, cognitiva, afectiva, instruccional, mediacional y ecológica. Mediante la determinación del significado institucional de referencia pretendemos analizar los conocimientos matemáticos relativos al contexto institucional donde fue realizado el proceso de instrucción de los futuros docentes, lo cual forma parte del análisis de la faceta epistémica del conocimiento del profesor de matemáticas. En esta faceta se engloban los diferentes conocimientos disciplinares que el profesor tiene o muestra sobre un contenido matemático específico (conocimiento común, especializado y ampliado).

Para el análisis del conocimiento didáctico de los futuros profesores, se contempla el análisis de las facetas cognitiva y afectiva identificándolas como parte del conocimiento del contenido con relación a los estudiantes, y el análisis de las facetas interaccional y mediacional identificándolas como parte del conocimiento del contenido con relación a la enseñanza.

El Modelo CDM propone cuatro niveles de estudio de este conocimiento. Los dos primeros que serán los utilizados para dar sustento a la investigación se muestran a continuación (Godino, 2009, p. 21):

1. Prácticas matemáticas y didácticas. 
2. Configuración de objetos y procesos (matemáticos y didácticos).

Con respecto al primer nivel de análisis, se considera el análisis de las prácticas matemáticas y didácticas llevadas a cabo por el futuro profesor, de tal manera que se describen las acciones que éste llevó a cabo para resolver las tareas matemáticas propuestas para lograr contextualizar los contenidos y promover los aprendizajes de sus alumnos. También se describe de manera general la actuación como docente.

Con respecto del segundo nivel, se centra el interés en los objetos matemáticos, de tal manera que se logran describir éstos y algunos procesos matemáticos que intervienen en la realización de las prácticas, así como los que emergen de ellas. La finalidad de este nivel es poder describir la complejidad de los objetos y prácticas matemáticas y didácticas como un factor explicativo de los conflictos que se producen en su realización.

Para los propósitos del trabajo los niveles de estudio mencionados anteriormente se eligen por contemplar dentro del análisis los elementos (prácticas matemáticas y didácticas) que nos llevarán a caracterizar el conocimiento didáctico-matemático de los sujetos de estudio.

Los constructos teóricos propuestos por el EOS que han sido considerados aquí, serán utilizados en la investigación para analizar, conocer y describir los conocimientos didácticos y disciplinares que los normalistas pusieron en juego durante su Fase de Prácticas.

\subsection{Aspectos metodológicos}

El trabajo de investigación que estamos desarrollando consiste en un estudio de carácter descriptivo de la Fase de Prácticas de futuros profesores de matemáticas de secundaria. La metodología que se pretende emplear se corresponde con los estudios de corte cualitativo y consta de: "Descripciones detalladas de situaciones, eventos, personas, interacciones y comportamientos que son observables, incorporando la voz de los participantes, sus experiencias, actitudes, creencias, pensamientos y reflexiones tal y como son expresados por ellos mismos" (Sandín, 2003, p.121), consistente con el marco teórico seleccionado.

Las acciones metodológicas que han sido realizadas se mencionan a continuación:

- Para la elección de los casos de estudio, se tomó en cuenta a los estudiantes de la Licenciatura en Educación Especialidad Matemáticas de ENSH que iniciaban su séptimo semestre, en el cual da inicio su Fase de Prácticas. Se eligieron a tres normalistas que desarrollarían su Fase de Prácticas con alumnos que iniciaban el tercer grado en una escuela secundaria pública de Hermosillo, Sonora. Los contenidos matemáticos que impartirían se ubican en los apartados "Patrones y ecuaciones" y "Figuras y cuerpos" del Bloque II del Programa de Matemáticas de Tercer Grado de Secundaria. Es importante señalar que los contenidos matemáticos estudiados fueron determinados en el momento que fue definida la institución de educación secundaria donde los normalistas desarrollarían su Fase de Prácticas.

- Determinación del significado institucional de referencia. 
- Elaboración de un formato de observación en el aula, que sería utilizado durante la observación de la Fase de Prácticas de los normalistas. Es importante resaltar que el formato de observación empleado fue diseñado para la observación del contenido matemático que los normalistas desarrollarían, esto con la intención de identificar aquellos elementos que en el Modelo CDM se identifican como parte del conocimiento didáctico-matemático del profesor, y que son puestos en juego durante el proceso de estudio.

- Observación en el aula de los temas correspondientes a los ejes "Sentido numérico y pensamiento algebraico" y "Forma, espacio y medida" correspondientes al Bloque II. De esta manera se identifica a los sujetos de estudio como Sujeto A, Sujeto B y Sujeto C. En los contenidos de "Patrones y ecuaciones" se observaron 7 sesiones del Sujeto A y 9 sesiones de los Sujetos B y C. Para los contenidos de "Figuras y cuerpos" fueron observadas 7 sesiones del Sujeto A y 8 sesiones de los Sujetos B y C.

- Toma de datos. Las sesiones observadas fueron 48, 25 correspondientes a "Patrones y ecuaciones" y 23 correspondientes a "Figuras y cuerpos" del Bloque II. La duración de las sesiones oficialmente fue de 45 minutos, pero podrá ser apreciado en análisis posteriores, que la clase efectiva promedia entre 35 y 40 minutos. En cada una de las sesiones observadas se utilizó audiograbadora y se empleó cámara de video, para registrar lo realizado durante el proceso de estudio en el aula.

En el trabajo de investigación que se está realizando se tiene contemplado presentar los resultados de la observación de las sesiones correspondientes a los contenidos de "Patrones y ecuaciones" del Bloque II.

Se pretende contrastar las acciones realizadas por los futuros docentes durante su Fase de Prácticas con las actividades planificadas para la clase, y con el significado institucional de referencia. Esto se hace con el propósito de analizar las diferentes facetas del conocimiento didáctico-matemático consideradas en la investigación como parte del Modelo CDM.

Las acciones metodológicas descritas son las que permitirán la descripción, análisis y caracterización del conocimiento didáctico-matemático de futuros profesores de matemáticas de secundaria en su último año de formación inicial.

\section{Avances}

En el trabajo de investigación que se reporta, interesa caracterizar el conocimiento didácticomatemático de los futuros docentes de secundaria en su periodo de Fase de Prácticas. Por esta razón, parte de nuestro análisis centra el interés en los sistemas de prácticas que la institución (donde se desarrolló la Fase de Prácticas) establece como referencia, por ende, también en los objetos matemáticos que intervienen y los que emergen de dichos sistemas.

En esta parte se muestra el análisis elaborado para la determinación del significado institucional de referencia sobre los contenidos matemáticos estudiados. Para esto, se toman en cuenta los sistemas de prácticas matemáticas identificados en el libro de texto de Matemáticas 
III (Ríos y Nebbia, 2013), planes de clase (SEP, 2011b) y Programa (SEP, 2011a), todos correspondientes a tercer grado de educación secundaria, específicamente los relacionados con el contenido: "Uso de ecuaciones cuadráticas para modelar situaciones y resolverlas usando la factorización".

Estos sistemas de prácticas conforman el significado institucional de referencia, que servirá para contrastar con los sistemas de prácticas matemáticas efectivamente desarrollados por los futuros docentes y caracterizar su conocimiento matemático.De este modo, es posible analizar la faceta epistémica del conocimiento didáctico-matemático del profesor, identificada en el Modelo CDM como aquella donde se engloban los diferentes conocimientos matemáticos relativos al contexto institucional donde se desarrolla el proceso de estudio (Godino, 2009).

4.1 Significado institucional de referencia sobre el contenido "Uso de ecuaciones cuadráticas para modelar situaciones y resolverlas usando la factorización"

Dado que el significado institucional de referencia de determinado objeto matemático está determinado por sistemas de prácticas, intervienen y emergen algunos de los seis tipos de objetos matemáticos primarios, así como combinaciones de los mismos. Consideramos de esta forma que a través de la identificación de éstos es posible determinar el significado institucional de referencia.

Con base en lo anterior, se presenta la configuración de objetos matemáticos primarios que componen el significado institucional de referencia sobre el contenido "Uso de ecuaciones cuadráticas para modelar situaciones y resolverlas usando la factorización". Esto se hace para identificar los sistemas de prácticas que la institución (escuela secundaria) establece como referencia, identificando de esta forma los conocimientos matemáticos relativos al contexto institucional donde se desarrolla el proceso de estudio.

En virtud de que el material revisado es extenso (planes de clase, libro de texto y programa) y presentarlo en su totalidad se muestra complicado, la identificación de los objetos matemáticos primarios y sus combinaciones se hace a partir de agrupamientos que toman como base los tipos de situaciones (o problemas) que aparecen en los documentos analizados.

Como resultado del análisis de los diferentes documentos que conforman el sistema de prácticas matemáticas que determinan el significado institucional de referencia se logra describir la forma en que son introducidos los contenidos; el papel que juegan los diferentes objetos matemáticos primarios identificados; y la relación entre los mismos (configuración epistémica). Lo anterior, se hace con el propósito de identificar las prácticas matemáticas que la institución promueve con relación a los contenidos de interés. 


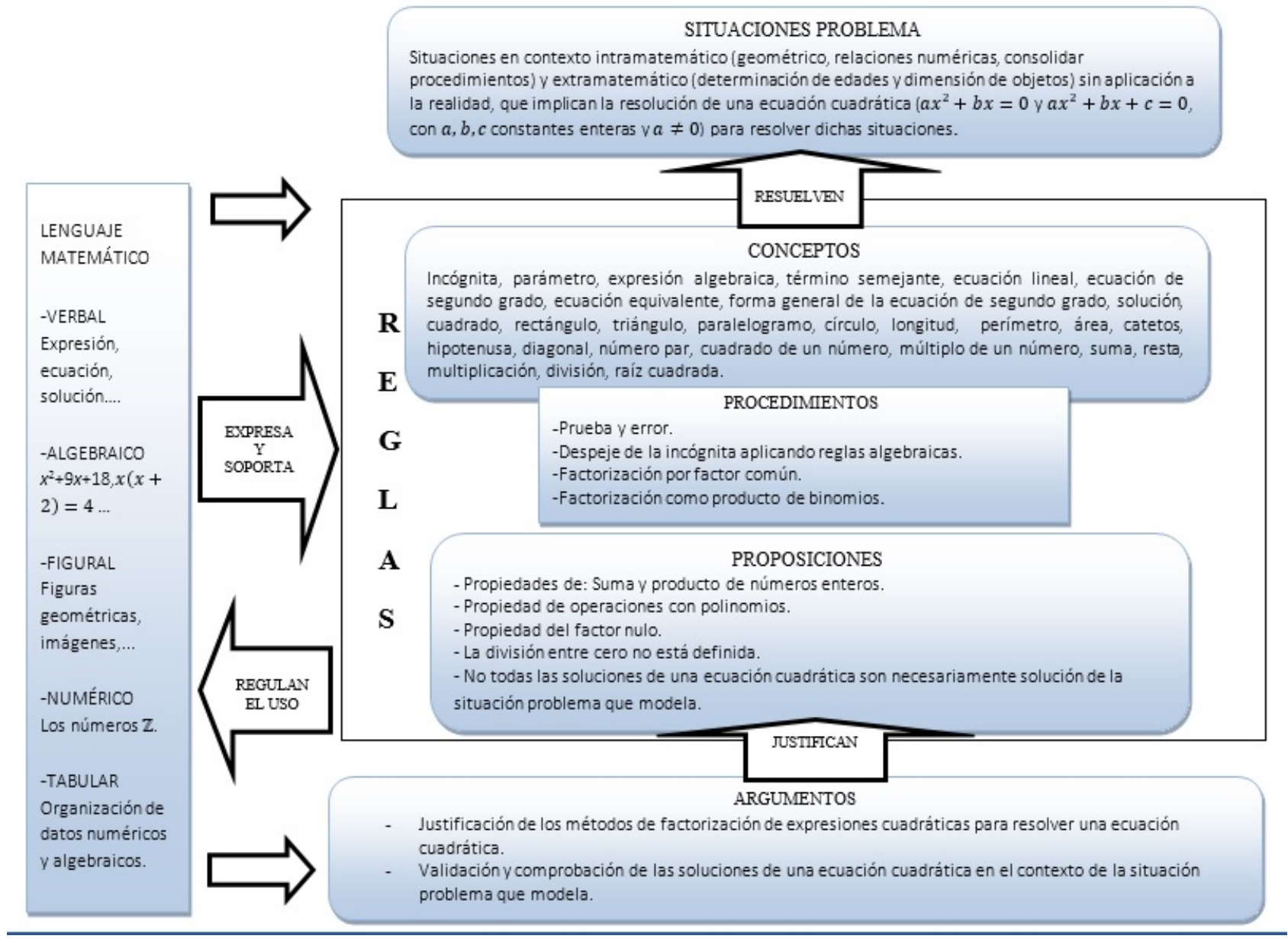

Figura 1: Configuración epistémica asociada al contenido "Uso de ecuaciones cuadráticas para modelar situaciones y resolverlas usando la factorización".

La configuración anterior permite describir la complejidad de los objetos matemáticos primarios y las prácticas matemáticas, como un factor explicativo de posibles conflictos en su realización. Este análisis ayudará en la descripción de los sistemas de prácticas matemáticas promovidas por los futuros profesores, desde su planeación para la clase hasta el momento de su implementación en el aula durante su Fase de Prácticas. Lo anterior será posible realizando un análisis de la actividad matemática y por ende de los conocimientos matemáticos involucrados.

\section{A modo de cierre}

Siendo el objetivo caracterizar el conocimiento didáctico y disciplinar que ponen en juego los sujetos de estudio durante su Fase de Prácticas, el análisis reportado en este artículo está enfocado a la caracterización del conocimiento disciplinar de referencia sobre un contenido específico. Aún hace falta llevar a cabo el análisis de los sistemas de prácticas matemáticas 
que los futuros profesores promovieron desde la planeación de los contenidos de clase hasta el momento de su implementación en el aula. De esta forma se pretende realizar un análisis de la actividad matemática realizada por los sujetos de estudio, que posteriormente llevará a conocer, describir y caracterizar los conocimientos matemáticos que pusieron en juego.

Lo anterior lleva al análisis de la faceta epistémica del Modelo CDM. Para el análisis de otras facetas relacionadas con los conocimientos didácticos como las facetas cognitiva y afectiva (el conocimiento del contenido con relación a los estudiantes) y las facetas interaccional

y mediacional (el conocimiento del contenido con relación a la enseñanza), se está llevando a cabo el análisis de la información recabada durante la observación de su Fase de Prácticas, guiando este análisis por medio de las pautas y criterios que el Modelo CDM establece. De esta forma se pretende caracterizar el conocimiento didáctico-matemático que futuros profesores de matemáticas ponen de manifiesto sobre un contenido específico, al encontrarse en su último año de formación inicial.

\section{Referencias}

[1] Godino, J. D.(2009). Categorías de análisis de los conocimientos del profesor de matemáticas. UNIÓN: Revista Iberoamericana de Educación Matemática, 20, 13-31.

[2] Godino, J. D., Batanero, C. y Font, V. (2009). Un Enfoque Ontosemiótico del conocimiento y la instrucción matemática. Versión ampliada y revisada al 8/Marzo/2009 del artículo Godino, J. D., Batanero, C. y Font, V. (2007). The onto-semiotic approach to Research in Mathematics educations. Zentralblatt fur Didaktik der mathematic (ZDM): The international journal on Mathematics Education, 39(1-2). 127-135.

[3] Hill, H. C., Ball, D. L. y Schilling, S. G.(2008). Unpacking pedagogical content knowledge: Conceptualizing and measuring teachers' topic-specific knowledge of students. Journal for Research in Mathematics Education, 39, 372-400.

[4] Ríos, D. y Nebbia, C. (2013). Fortalezco mis competencias. Matemáticas 3 (1ra. ed.), SM de ediciones, México, D.F..

[5] Sandín, M.(2003). Investigación cualitativa en Educación, Fundamentos y Tradiciones. McGraw-Hill/ Interamericana de España, Madrid, España.

[6] Sandoval, E. F.(2009). La inserción a la docencia. Aprendiendo a ser maestro en México. Revista de currículum y formación de profesorado, 13(1), 184-194.

[7] Schoenfeld, A. H. y Kilpatrick, J.(2008). Towards a theory of proficiency in teaching mathematics. En D. Tirosh y T. Wood (eds.), Tools and Processes in Mathematics Teacher Education, 321-354, Sense Publishers, Rotterdam.

[8] Secretaría de Educación Pública. (2011a). Programas de estudio 2011: Guía para el maestro, SEP, México. 
[9] Secretaría de Educación Pública. (2011b). Planes de clase de matemáticas para tercer grado de secundaria, SEP, México. Recuperado el 10 de junio de 2013, en http:www . curriculobasica.sep.gob.mx/index.php/sec-mat-tercer-grado?sid=748.

[10] Shulman, L. S. (1987). Knowledge and teaching: Foundations of the new reform. Harvard Educational Review, 57(1), 1-22. 